\title{
N-Tuple S\&P 500 Index Patterns Across Decades, 1950s to 2011
}

Anastasios G. Malliaris

Loyola University Chicago, tmallia@luc.edu

Mary Malliaris

Loyola University Chicago

Follow this and additional works at: https://ecommons.luc.edu/business_facpubs

Part of the Business Commons

\section{Author Manuscript}

This is a pre-publication author manuscript of the final, published article.

\section{Recommended Citation}

Malliaris, Anastasios G. and Malliaris, Mary. N-Tuple S\&P 500 Index Patterns Across Decades, 1950s to 2011. Central European Journal of Operations Research, 22, 2: 339-353, 2014. Retrieved from Loyola eCommons, School of Business: Faculty Publications and Other Works, http://dx.doi.org/10.1007/ s10100-013-0298-3

This Article is brought to you for free and open access by the Faculty Publications and Other Works by Department at Loyola eCommons. It has been accepted for inclusion in School of Business: Faculty Publications and Other Works by an authorized administrator of Loyola eCommons. For more information, please contact ecommons@luc.edu.

\section{(c) $($ () $\ominus$}

This work is licensed under a Creative Commons Attribution-Noncommercial-No Derivative Works 3.0 License. (C) Springer International Publishing AG 2014 


\title{
N-tuple S\&P 500 index patterns across decades, 1950s to 2011
}

\author{
A.G. Malliaris \\ Departments of Economics and Finance \\ Loyola University Chicago \\ 1 E. Pearson, Chicago, IL 60611 \\ tmallia@luc.edu \\ and \\ Mary Malliaris \\ Department of Information Systems \& Operations Management \\ Loyola University Chicago \\ 1 E. Pearson, Chicago, IL 60611 \\ mmallia@luc.edu
}

\begin{abstract}
Numerous studies have analyzed the movements of the S\&P 500 Index using several methodologies such as technical analysis, econometric modeling, time series techniques and theories from behavioral finance. In this paper we take a novel approach. We use daily closing prices for the S\&P 500 Index for a very long period from 1/3/1950 to 7/19/2011 for a total of 15,488 daily observations. We then investigate the up and down movements and their combinations for 1 to 7 days giving us multiple possible patterns for over six decades. Some patterns of each type are more dominant across decades. We split the data into training and validation sets and then select the dominant patterns to build conditional forecasts in several ways, including using a decision tree methodology. The best model is correct $51 \%$ of the time on the validation set when forecasting a down day, and $61 \%$ when forecasting an up day. We show that certain conditional forecasts outperform the unconditional random walk model.
\end{abstract}

Keywords: S\&P 500 Index; Patterns across decades; Random walk; Decision tree methodology JEL: C44, C53, G14, G17 


\section{N-tuple S\&P 500 index patterns across decades, 1950s to 2011}

\section{Introduction}

A variety of different models are used in predicting returns on the S\&P 500 stock index. These models use significantly different methods for determining buy and sell signals on either individual stocks or market index futures. The signals are typically either technical indicators such as a moving average, or momentum, or micro market or macro specific fundamental indicators, such as dividend yield, interest rates, (for market specific indicators) or economic growth (macro specific).

The aim of this paper is to investigate sequences of Up and Down movements of the S\&P 500 Index using a novel approach. To study the daily movements of the Index and its patterns for 1 up to 5 days, we carefully record all actual such sequences obtained from a portion of our total sample and the frequency of each pattern is calculated. Then these results are translated into a decision-making strategy in an attempt to apply the patterns obtained to new data from a validation set and derive conditional forecasts.

In section 2, we offer a selective review of the related literature and in section 3 we discuss our sample and how it is divided into nonoverlapping training and validation sets. Then we collect the results and analysis from the training set and present them in section 4 in the form of tables for ease of understanding. The decision making strategy is presented in section 5 and the forecasting performance along with its analysis are given in section 6. A summary concludes the paper in section 7 .

\section{Review of the literature}

From the early 1970s, a major dichotomy has persisted in the literature on the behavior of stock prices between market efficiency theorists and active portfolio management advocates. The theory of market efficiency has argued that because information is valuable it is not wasted or ignored and market participants move rapidly to evaluate and trade using all available information, thus contributing to the formation of prices that fully reflect such information. As new information becomes available, sometimes positive, others negative, often significant while at other times only mildly important, it also becomes quickly incorporated into prices. Prices 
then follow random walks in view of the randomness of information. It is well known that the market efficiency hypothesis is the most empirically tested theory in finance and these tests have extended significant support. An immediate corollary of market efficiency and the random walk behavior of prices is that prices cannot be predicted, since they are driven by the arrival of new public information.

In contrast to the teachings of market efficiency about the futility of searching for methods to beat the market, a large number of individual investors and fund managers tirelessly try to outperform the market. There is no doubt that such a task is very difficult and there are only few superstars, such as George Soros or Warren Buffett, who have outperformed the market over few decades. Thus, while market efficiency remains the dominant theory about financial markets, considerable effort is exerted daily by professionals to outperform the market. These efforts have also been extended to academics that almost simultaneously with the early developments of market efficiency have been searching for active management strategies. The dichotomy then continues today between market efficiency that remains the ordained theory, yet to be replaced, and a collection of methods and strategies that search for ways to outperform the market or simply to demonstrate that markets deviate from perfect efficiency and rationality. There are several groupings of this research such as technical analysis, econometric methods and behavioral finance each with an enormous set of papers and a large variety of conclusions.

An eclectic sample of studies that investigate the effectiveness of technical analysis and active management strategies includes: Fama and Blume (1966), Jensen and Benington (1970), Brown and Jennings (1989), Brock, Lakonishok and LeBaron (1992), Blume, Easley and O’Hara (1994), Gencay (1998), Allen and Karjalainen (1999), Lo, Mamaysky and Wang (2000). Some of these papers are critical of simple technical rules and demonstrate than even simple random walks often generate trading rules that may temporarily give successful trading signals that appear to predict well; other papers show that once transactions costs are included the predictive advantage of trading rules is diminished or completely eliminated; and finally, some find evidence that some technical indicators have significant predictive ability.

More recently, Schulmeister (2007) looks at technical trading strategies on the S\&P500 futures and their ability at predicting returns. This paper finds that in the 1960s and 1970s the use of daily stock data was profitable among strategies generating a buy-sell decision based on the futures price relative to its moving average Specifically in the paper, the author uses a short run 
and long run moving average, and going long when the short term moving average goes above the long run moving average, and short selling when the opposite occurs. However, testing the same indicators from 2000-2006 saw diminishing results for those strategies. One possible explanation given by the author is that there is likely a trend to higher frequency in trading on technical indicators and that now, 30 minute data is insufficient to produce a profitable strategy.

Other papers focus more on fundamental aspects of stocks or the S\&P index, as well as macroeconomic data when developing econometric return forecasting model. Doran, Ronn, Goldberg (2005) (DRG) examine return predictability using more fundamental variables. In developing their model, the authors utilize two equations used to estimate required return; a dividend growth rate model and the Sharpe security market line. Since they were working with the S\&P Index and not with individual stocks, the authors used the VIX as a proxy for stock volatility. The results of their study were that short term expected returns were highly volatile, largely due to changes in VIX. One of the inherent issues in their model, which they address, is the estimation of market risk premium in their model. The market risk premium did go negative on two occasions. The two occasions were in 1987, right before the crash, and in 2000 during the tech bubble. As a result, they determine that those levels of market risk premium could be attributed to irrational exuberance.

Other studies, such as Avramov and Chordia (2002) examine work done with regard to how firm specific factors are used to predict return. Notable factors that could be applied to S\&P returns are the Treasury yield, and dividend yield. They also find that there are many other firm specific factors that hold up to out of sample testing of return predictability. However, this predictability holds best for small-cap stocks, growth stocks, and momentum stocks, and not the broader market. Other findings of the paper are that using the equity risk premium allows for negligible out of sample predictability, causing the authors to conclude that firm specific factors such as dividend yield and treasury yields are more useful at predicting returns.

Beyond technical and econometric analysis behavioral finance has emerged the last 30 years as a flourishing collection of theories that challenge market efficiency. Behavioral finance accepts minor deviations from rationality and perfect arbitrage, introduces asymmetric utility describing gains versus losses, explains overvaluation, herding, momentum, feedback and various other characteristics of trading activities and thus encourages the search for a realistic understanding of asset pricing. A detailed survey of the leading contributions is presented in 
Hirshleifer and Subrahmanyan (1998) and contributions by Barberis, Shleifer and Vishny (1998), Hong and Stein (1999).

DeLong, Shleifer, Summers and Waldman (1990) study the impact of noisy traders and show that if they follow positive feedback strategies, that is, if noisy traders buy when prices rise and sell when prices fall, then it may pay for traders to buy ahead of noise demand and to also sell before noise traders. Further examining the behavior of noise traders, Cutler, Poterba and Summers (1991) demonstrate that returns are positively serially correlated at high frequency and negatively correlated over long horizons. McInish and Wood (1992) examine high frequency minute-by-minute data and confirm the presence of certain consistent patterns. Intraday patterns are also investigated by Chan and Karolyi who find evidence of strong intermarket dependence in the volatility of the stock market index and the stock index futures.

Recently, Baur, Dimpfl and Jung (2012) revisit return autocorrelations and find for daily data over thirty years that lower quantiles autoregressions exhibit positive dependence on past returns in contrast to upper quantiles are marked by negative dependence.

The main conclusion of this rapid review of selected studies searching for stock index patterns demonstrates that this is an area of active and challenging research. In this paper we take a novel approach. We use daily closing prices for the S\&P 500 Index for a very long period from $1 / 3 / 1950$ to $7 / 19 / 2011$ for a total of 15,488 daily observations. We then search and record UP (U) and DOWN (D) movements for 1 day, combinations of $U$ and Down movements (UU, UD, DU and DD) for 2 days. Similarly, patterns were tabulated for 3, 4, 5, and 6 day strings. Following a discussion of patterns over six decades, we divide the data set into training and validation sets, then proceed to use this information to develop and compare a variety of forecasts. Section 3 details and discusses the some of these patterns. In section 4 , we develop and compare forecasts from the patterns themselves, with the random walk, and using a data mining decision tree algorithm. Section 5 contains conclusions from the approaches, and recommendations for future research.

\section{Multi-decade patterns}

Patterns of up and down movement were tallied in two ways. In the first set of charts, we look at the individual string patterns of Up and Down for one, two, three, and four days. Though we also track the five and six day pattern strings, their graphs are too complex to display here. 
Multi-day strings are calculated by concatenating single day strings. Thus, if we have, for a single week, the following single day movements: U, D, D, U, U, we would have the three-day pattern UDD associated with Wednesday, DDU with Thursday, and DUU with Friday.

We then reconfigure this information by counting only the number of Up days. Thus, for example, UD and DU each have 1 Up day and are collapsed into one category. This second way of counting allows us to focus on the number of positive movements that occur in strings of a specific length while suppressing exactly where those occur. Looking at the following figures showing up and down movement, we see the following result that, contrary to the random walk hypothesis where stocks move randomly, our first tabulation of actual up and down movements across six decades confirms that the S\&P 500 consistently moves up in the long term, as seen in Figure 1. In these figures, each type of string is displayed as a bar, with the decade shown below the set of bars. Pattern occurrence is shown on the y-axis as the percent of times it appeared in that decade. The last decade contains data only from years 2010 and 2011, but the percent display allows us to easily compare what is happening at the beginning of this decade with the other decades.

Fig 1 Percent of Up [U] and Down [D] movements per decade, 1950 through 2011

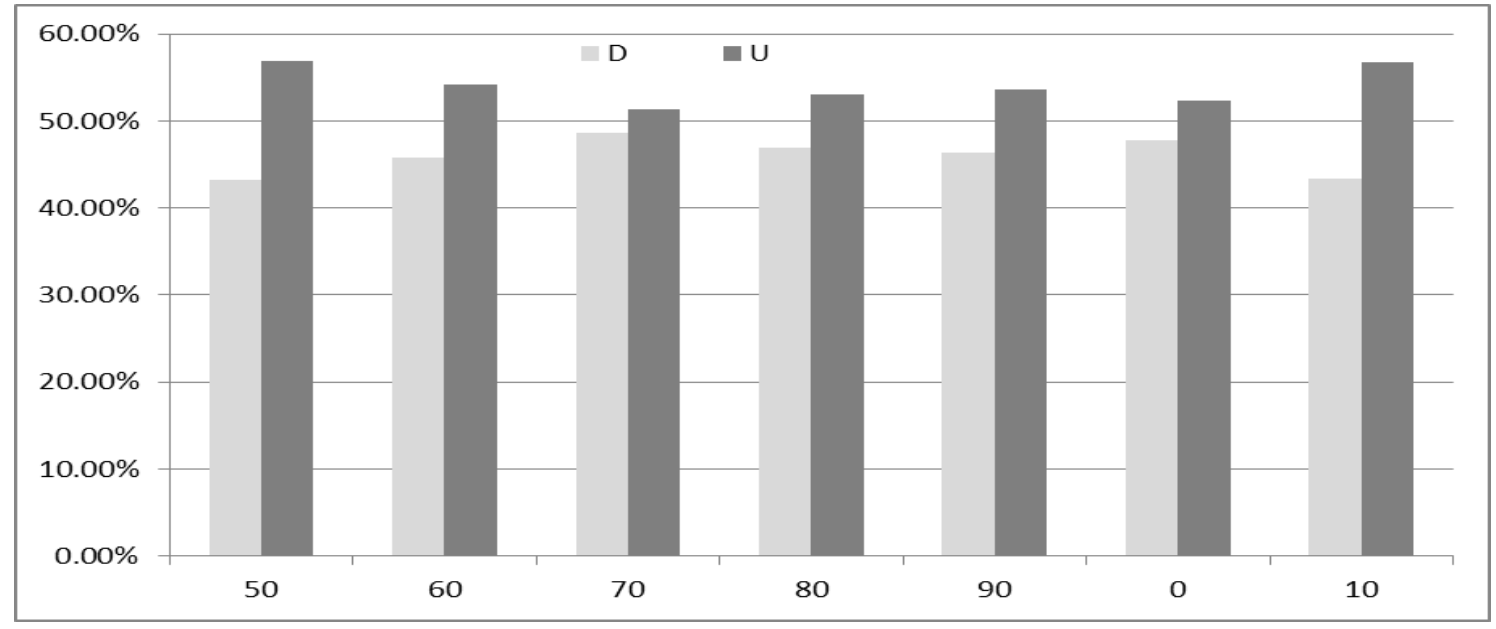

The second result of two-day strings, shown in Figure 2, indicates that from 1950 until 2000, upup was the most dominant pattern in two-day movements. We also notice that, in the single decade from 2000 to 2009, it is no longer the case that up-up dominates. The two center 
columns (up-down and down-up) dominate in this period, emphasizing the frequent day to day change in movement of the S\&P during that decade.

Fig 2 The four two-day strings of Up and Down across decades

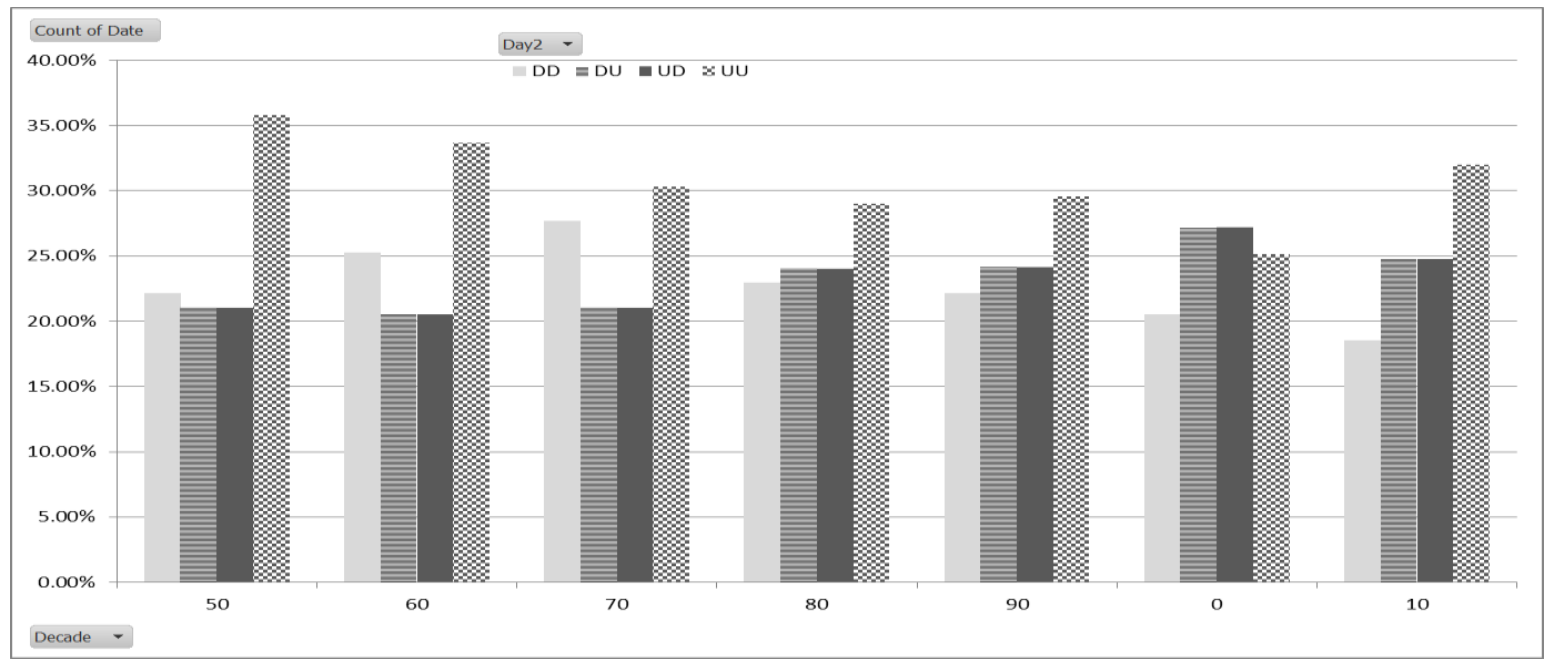

When it comes to three and four day strings of Up and Down patterns, all ups dominate in most, but not all, of the decades. Figure 3 shows these three-day patterns as percent of occurrence. UUU, the grey bar at the right side of each group, is highest in the 1950s, then decreases across the decades, with a low during the decade from 2000 through 2009. As we move from 1950 to 2010, we see that fewer patterns dominate and there is more even distribution across possible patterns. Then, during 2010 and 2011, we see the initial part of this last decade returning to a greater emphasis on up movement. There is more stability in DDU and UDD across decades.

Fig 3 Three-day strings of Up and Down movement across decades 


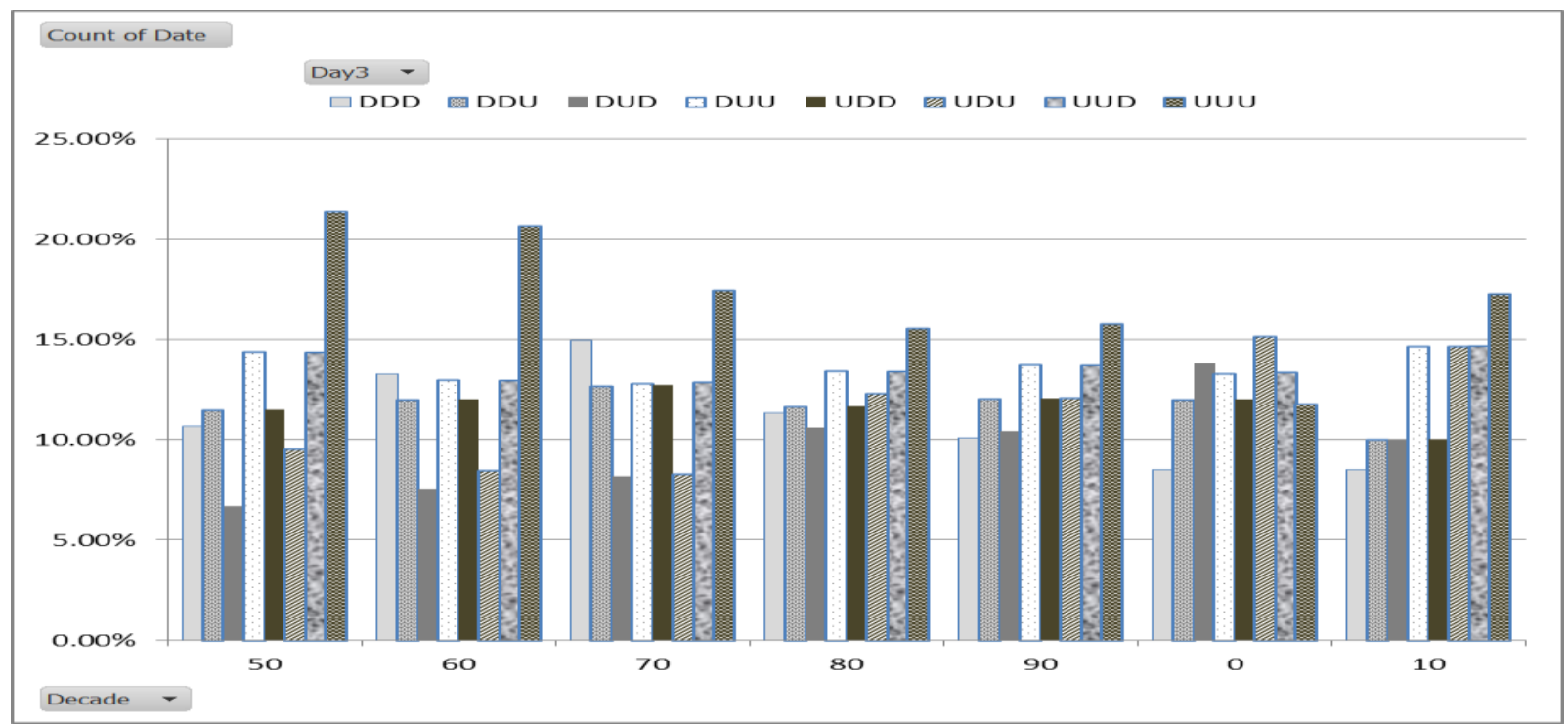

In Figure 4, we have a total of 16 possible patterns, and each one of them occurs in the dataset. Notice that the percent of times that UUUU occurs is highest early on, then decreases over the decades. UDUU and UUDU occur about the same amount of time. In the 50s, 60s, and 70s, DUDU and UDUD were the least occurring patterns. This set of patterns changes in the 80s where the daily change becomes more likely, and remains true. We also notice that the leftmost bar, representing the string of four down days in a row, decreases over time as a percent of occurrence. As the number of days in the pattern increases, these charts become more congested, so we will not display the bar charts for five and six day patterns. Instead, we will switch to looking at this data in a slightly altered fashion.

Fig 4 Four day strings of Up and Down movement across decades 


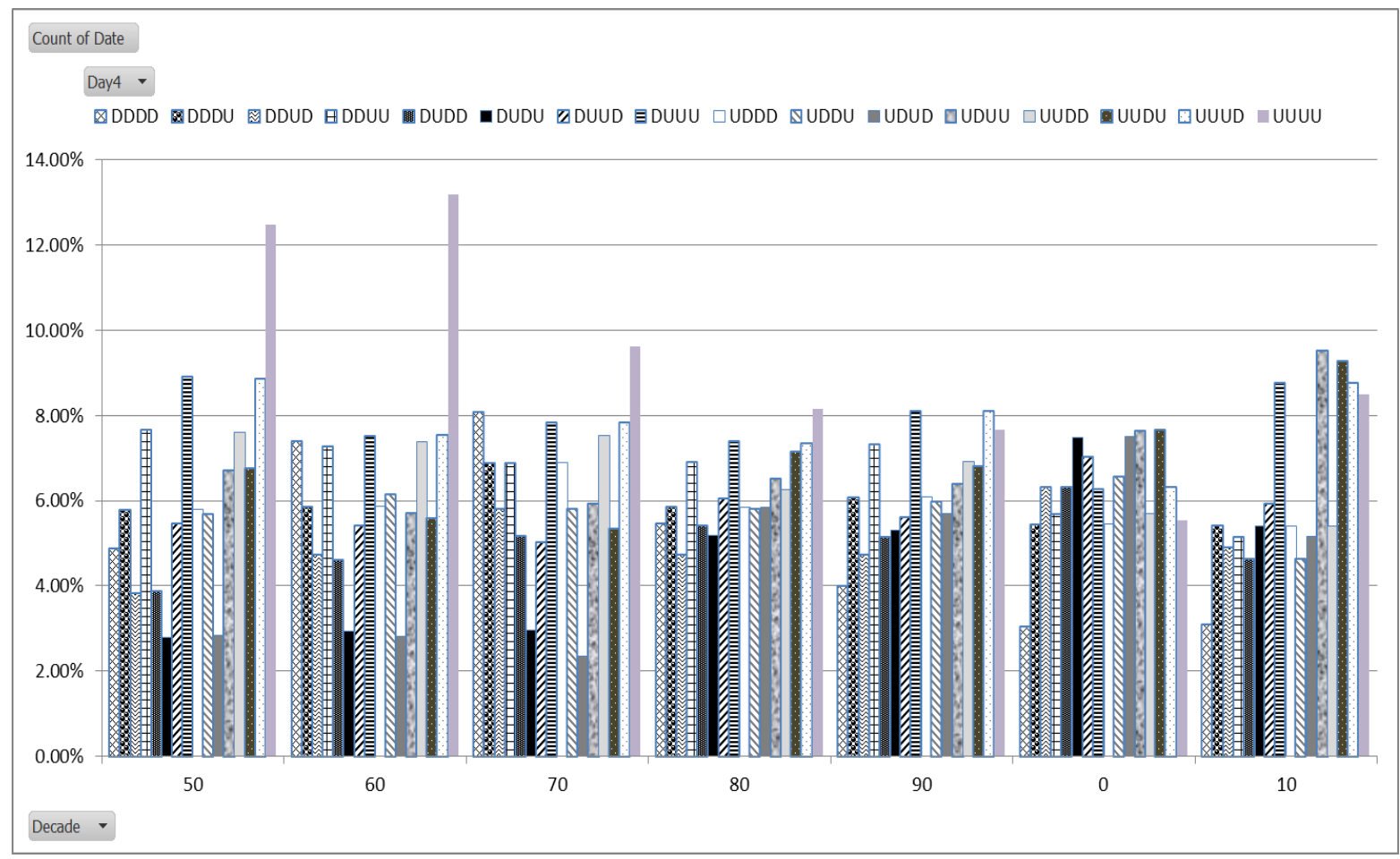

Another way to display this data is by focusing on only the number of up movements in strings of a given length. For example, as seen earlier when we look at only one day across decades, up occurs more than down every decade. Looking at two-day strings, whereas we originally had four patterns, this revised focus gives us only three. That is, we can have 0,1 or 2 possible ups. When we have a string of DD, this yields $0 \mathrm{Ups}$; strings of the form DU or UD are both examples of exactly $1 \mathrm{Up}$; and UU gives us 2 Ups. Figure 5 displays these counts. As with the previous graphs, all numbers are displayed as the percent of occurrence during the decade. We see that having a single up in a string of two days is most likely in any decade. The frequency of two ups in any two-day string decreases across decades until the current one where it is showing an upward trend. We also see that, in every decade, two Ups occurred more than zero Ups in each time block.

Fig 5 Percent of 0,1 , and 2 Up movements in 2 days 


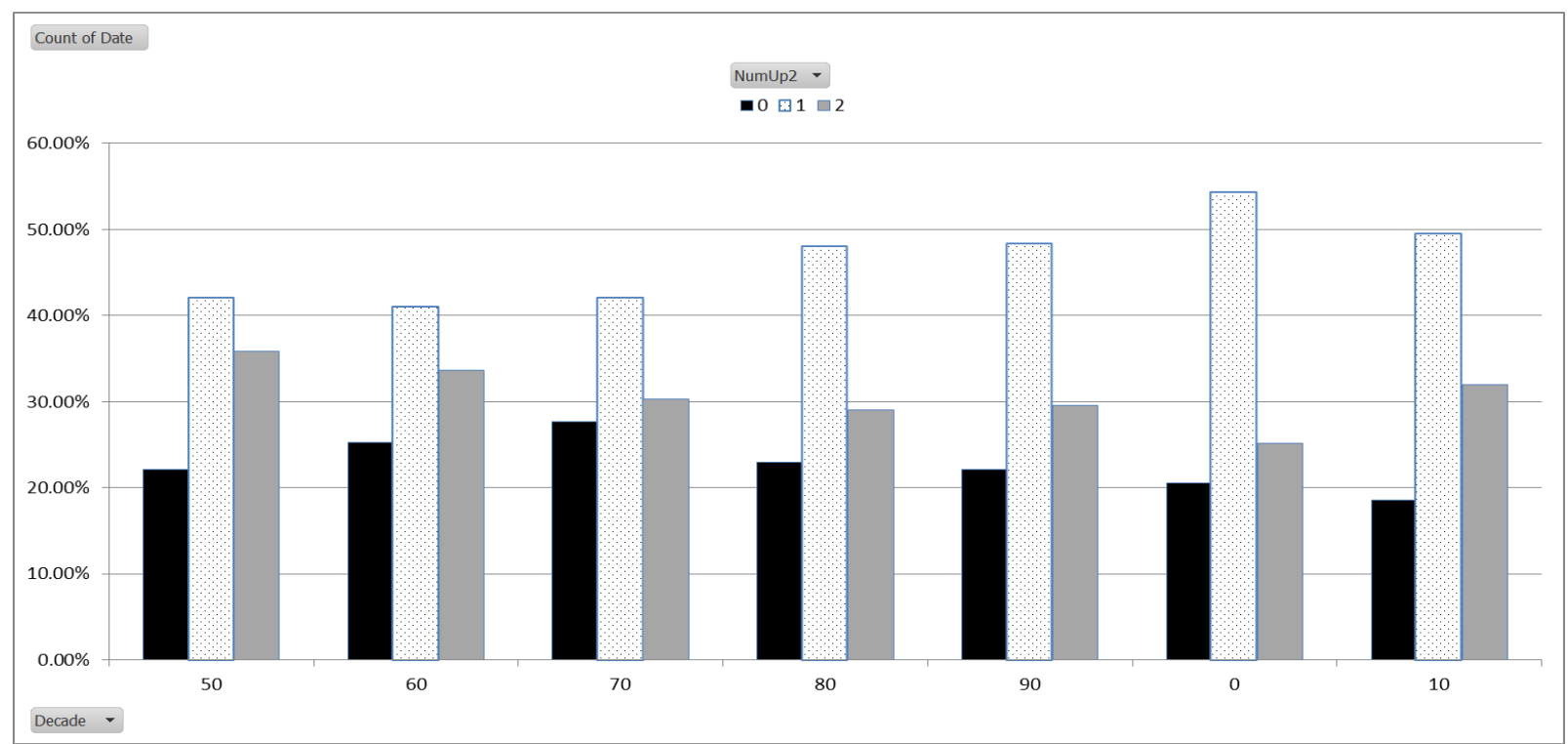

For three and four day strings, you are most likely to have exactly two Ups. Figure 6 shows that, for three-day strings, there are more $2 \mathrm{~s}$ in every decade, fewer $0 \mathrm{~s}$ than $3 \mathrm{~s}$, and fewer $1 \mathrm{~s}$ than $2 \mathrm{~s}$.

Fig 6 Percent of 0, 1, 2, and 3 Up movements in 3 days

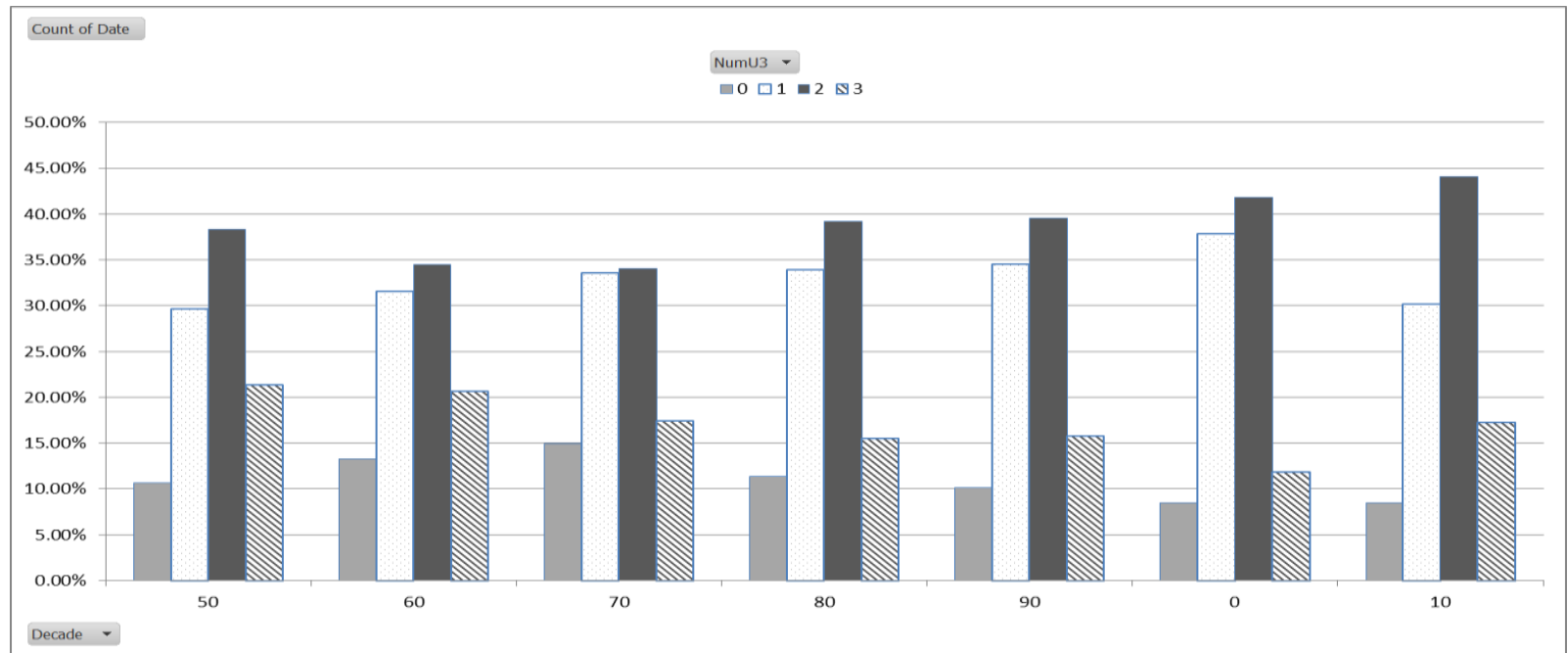

In Figure 7, for 4-day strings, we see the peak for most decades comes with exactly 2 days out of 4 having Up movements. However, in every case, there are more $3 \mathrm{~s}$ than $1 \mathrm{~s}$, and more $4 \mathrm{~s}$ than $0 \mathrm{~s}$. So, when we extend our view to four days at a time, there are very few strings of four Down days.

Fig 7 Percent of 0, 1, 2, 3 and 4 Up movements in four days 


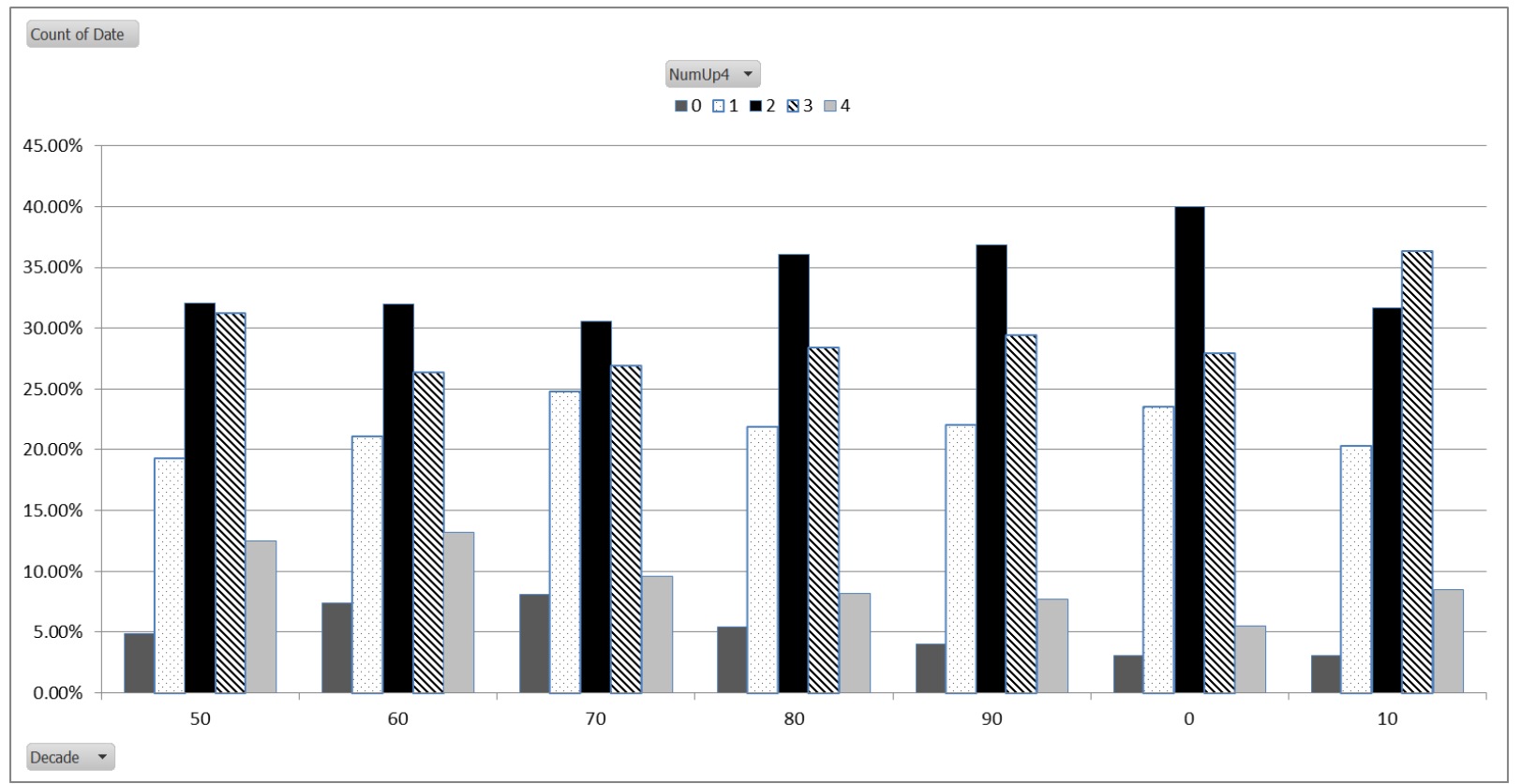

In the last chart that we will display, we shown the percent count of the number of times that Up occurred in the five-day strings. Figure 8 indicates that, in every decade, we see the following: More $3 \mathrm{~s}$ (shown as the darkest bar in the center) than $2 \mathrm{~s}$, more $4 \mathrm{~s}$ than $1 \mathrm{~s}$, and more $5 \mathrm{~s}$ than $0 \mathrm{~s}$.

Fig 8 Percent of $0,1,2,3,4$, and 5 Up movements in 5 day strings

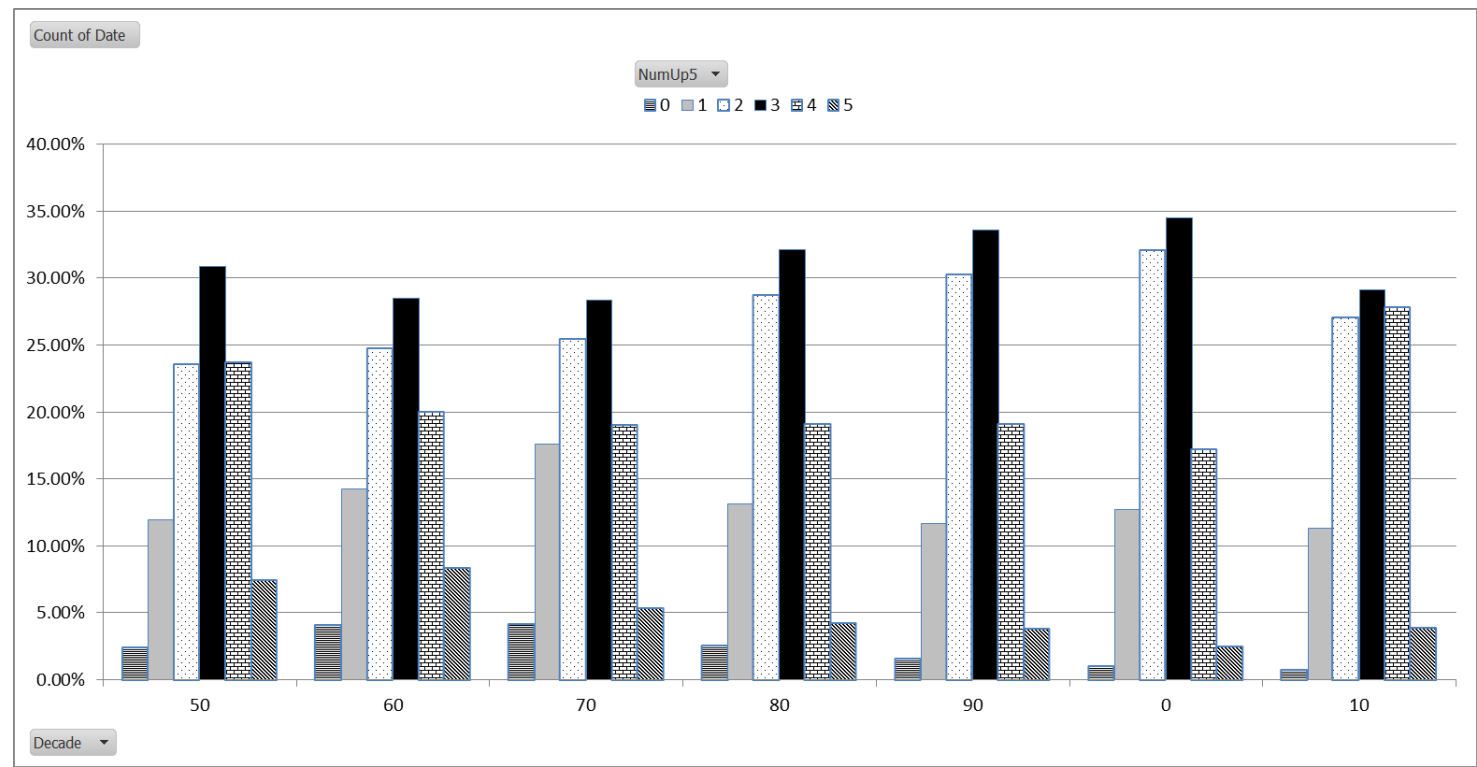


This series of figures illustrates that some patterns typically occur more across every decade, even though there are shifts over time. In the next section, we investigate whether or not we can use the similarities over time to develop useful forecasts.

\section{Forecasting}

To translate this analysis into a decision-making strategy to attempt to predict up or down movement tomorrow conditional on the past patterns from 1 to 7 days, we separated the total sample into two parts, a training set and a validation set. The training set comprised all data from January 1950 through December 2009 [15,087 rows]. The validation set used the data from January 2010 through July 2011 [387 rows]. The training set was used to determine the most dominant patterns with all strings of a given length. These dominant patterns were then used to determine the direction of the forecasts for each day in the validation set.

With the training data set, we calculated the number of times each one to seven-day pattern of Ups and Downs occurred. A sample of the patterns and the number of times each sample occurred in the set of 15,087 rows from January 1950 through December 2009 is shown in Table 1. We investigated patterns up to length 10, but past seven days, every possible pattern does not occur in the data set, making the comparison of pairs of patterns differing only in the last day un-doable. Thus, we used only string patterns of 1 to seven days.

Table 1 Example patterns and count of occurrence

\begin{tabular}{|l|r|l|l|r|l|l|r|}
\hline Pattern & Count & & Pattern & Count & & Pattern & Count \\
\hline D & 7009 & & DDDDDD & 185 & & DDDDDDD & 83 \\
\hline U & 8078 & & DDDDDU & 215 & & DDDDDDU & 102 \\
\hline DD & 3539 & & DDDDUD & 179 & & DDDDDUD & 91 \\
\hline DU & 3469 & & DDDDUU & 247 & & DDDDDUU & 124 \\
\hline UD & 3470 & & DDDUDD & 220 & & DDDDUDD & 104 \\
\hline UU & 4609 & & DDDUDU & 168 & & DDDDUDU & 75 \\
\hline DDD & 1730 & & DDDUUD & 233 & & DDDDUUD & 116 \\
\hline DDU & 1809 & & DDDUUU & 283 & & DDDDUUU & 131 \\
\hline DUD & 1441 & & DDUDDD & 220 & & DDDUDDD & 111 \\
\hline DUU & 2029 & & DDUDDU & 209 & & DDDUDDU & 109 \\
\hline UDD & 1809 & & DDUDUD & 127 & & DDDUDUD & 61 \\
\hline
\end{tabular}




\begin{tabular}{|l|r|l|l|r|r|r|r|} 
UDU & 1660 & & DDUDUU & 204 & & DDDUDUU & 107 \\
\hline UUD & 2029 & & DDUUDD & 254 & & DDDUUDD & 133 \\
\hline UUU & 2580 & & DDUUDU & 203 & & DDDUUDU & 100 \\
\hline DDDD & 826 & & DDUUUD & 259 & & DDDUUUD & 124 \\
\hline DDDU & 904 & & DDUUUU & 334 & & DDDUUUU & 159 \\
\hline DDUD & 760 & & DUDDDD & 178 & & DDUDDDD & 103 \\
\hline DDUU & 1050 & & DUDDDU & 197 & & DDUDDDU & 117 \\
\hline DUDD & 769 & & DUDDUD & 158 & & DDUDDUD & 75 \\
\hline DUDU & 671 & & DUDDUU & 237 & & DDUDDUU & 134 \\
\hline DUUD & 871 & & DUDUDD & 133 & & DDUDUDD & 66 \\
\hline DUUU & 1158 & & DUDUDU & 140 & & DDUDUDU & 61 \\
\hline UDDD & 904 & & DUDUUD & 174 & & DDUDUUD & 91 \\
\hline UDDU & 905 & & DUDUUU & 224 & & DDUDUUU & 113 \\
\hline UDUD & 681 & & DUUDDD & 246 & & DDUUDDD & 138 \\
\hline UDUU & 979 & & DUUDDU & 212 & & DDUUDDU & 116 \\
\hline UUDD & 1040 & & DUUDUD & 181 & & DDUUDUD & 82 \\
\hline UUDU & 989 & & DUUDUU & 232 & & DDUUDUU & 121 \\
\hline UUUD & 1158 & & DUUUDD & 265 & & DDUUUDD & 131 \\
\hline UUUU & 1422 & & DUUUDU & 267 & & DDUUUDU & 128 \\
\hline DDDDD & 400 & & DUUUUD & 277 & & DDUUUUD & 139 \\
\hline DDDDU & 426 & & DUUUUU & 349 & & DDUUUUU & 195 \\
\hline DDDUD & 388 & & UDDDDD & 215 & & DUDDDDD & 90 \\
\hline
\end{tabular}

For each pair of patterns that differed in only the last movement, the pattern that occurred most often was specified as the dominant one of the pair. This pair-wise dominance formed the basis for forecasting on the validation set. Specifically, in the validation set, we made a series of seven predictions in the following way: For each of the patterns of days T-6 to T [i.e., seven day information], through $\mathrm{T}$ [one day information], a forecast for $\mathrm{T}+1$ was generated based on the percent of time that pattern had occurred in the training set.

For example, the first forecast, using only one day, would always predict Up tomorrow in the validation set because Up (8078 occurrences) dominated Down (7009 occurrences) in the training set in the one-day comparison of possible values. In two day forecasts, we have two cases to consider, those beginning with $U$ and those beginning with $D$. The strings beginning with U include UD and UU. Of these two, UD occurred in the training set 3470 times while UU occurred 4609 times. Since UU happened more often than UD, when we have a single input of $\mathrm{U}$ in the validation set, we will forecast that the second day would also be U. With the days where D occurred first in the string of two days, we could have either DD or DU. The training 
set indicates that DD occurred 3539 times and DU occurred 3469 times. In this case, since DD occurs more than DU, when D is the first day of a two-day string in the validation set, we will forecast that the second day in the string will also be D. In one example of a three-day string in the validation set, suppose that we currently know the string DDU has occurred. Data from the training set tells us that, of the two four-day possibilities that begin with DDU, we see that DDUU (that is, tomorrow Up) is more likely to occur than DDUD. So, for tomorrow, we will forecast Up in the validation set when the three days before tomorrow have the pattern DDU. Table 2 illustrates this using 4 and 3 day patterns from the training and validation sets respectively.

Table 2 Pattern examples for forecasting shows DDUU is more likely to occur than DDUD

\begin{tabular}{|l|c|c|c|}
\hline $\begin{array}{l}\text { Training Set } \\
\text { 4-day pattern }\end{array}$ & $\begin{array}{c}\text { Training Set } \\
\text { Count }\end{array}$ & $\begin{array}{c}\text { Validation Set } \\
\text { 3-day Pattern }\end{array}$ & $\begin{array}{c}\text { Validation Set } \\
\text { Forecast for the } 4^{\text {th }} \text { day }\end{array}$ \\
\hline$\underline{\text { DDUD }}$ & 760 & DDU & U \\
\hline$\underline{\text { DDUU }}$ & $\mathbf{1 0 5 0}$ & DDU & U \\
\hline
\end{tabular}

Thus, for every pair of strings that differ in the last letter of the pattern, the pattern for the next day that occurred most often in the training set was selected as the most likely behavior in the validation set. This method allowed us to generate seven forecasts for each row in the validation set. The first forecast used only 1 day strings, while the last used only information from the training set seven-day strings.

In addition, a random number was generated for each day of the validation data set; if the value was less than .5 then Down was predicted, otherwise the forecast was Up. We generated this random forecast to compare with the performance the n-tuple forecasts.

Then, for each of these eight forecasts on the validation set, we compared the value predicted with the actual value that occurred on day $\mathrm{T}+1$ and tallied the number of correct and incorrect calls. The number of correct predictions and the percent of time they were correct are shown in Table 3. We see that the most correct forecasts for tomorrow came from using the forecast from the current day. This is the least complex of the forecasts because Up dominates every year, so a prediction of Up for each day will be more correct than a prediction of Down for 
each day. However, this value with little information is very close to the prediction using six days of information, which does distinguish between the Up and Down days.

Table 3 Percent of time the validation set forecast was correct using 1 to 7 day patterns

\begin{tabular}{|c|c|c|c|c|c|c|c|}
\hline T-6 to T & T-5 to T & T-4 to T & T-3 to T & T-2 to T & T-1 to T & T & Random \\
\hline 212 & 218 & 212 & 205 & 205 & 195 & 219 & 178 \\
\hline $54.8 \%$ & $\mathbf{5 6 . 3 \%}$ & $54.8 \%$ & $53.0 \%$ & $53.0 \%$ & $50.4 \%$ & $\mathbf{5 6 . 6 \%}$ & $46.0 \%$ \\
\hline
\end{tabular}

Drilling down to the direction of each correct and incorrect forecast (correct forecasts shown in bold) in Table 4, we see that the weakness in every prediction strategy comes in the Down forecasts. Each D column lists the number of times Down was forecast when it was actually Down and when it was actually Up. In each case, the number of incorrect Down forecasts is greater than the number of correct Down forecasts. For the Up forecasts, the number of correct days is greater than the number of incorrect days for each method. Thus, the simple forecast of predicting Up always will be greater than the simple random walk 50/50, but is always wrong on Down days. The forecast using six day strings is correct on the Up days over $58 \%$ of the time, and correct on the Down days half of the time, so overall it is a better choice.

Table 4 Correct and Incorrect Up and Down forecasts

\begin{tabular}{|c|c|c|c|c|c|c|c|c|c|c|c|c|c|c|c|c|}
\hline \multirow{3}{*}{$\begin{array}{l}\text { Actual T+1 } \\
\text { Movement }\end{array}$} & \multicolumn{16}{|c|}{ Predicted Movement for $\mathbf{T}+1$} \\
\hline & \multicolumn{2}{|c|}{ T-6 to $\mathrm{T}$} & \multicolumn{2}{|c|}{ T-5 to $\mathbf{T}$} & \multicolumn{2}{|c|}{ T-4 to $\mathrm{T}$} & \multicolumn{2}{|c|}{ T-3 to $\mathbf{T}$} & \multicolumn{2}{|c|}{ T-2 to $\mathbf{T}$} & \multicolumn{2}{|c|}{ T-1 to $\mathbf{T}$} & \multicolumn{2}{|c|}{$\mathbf{T}$} & \multicolumn{2}{|c|}{ Random } \\
\hline & D & $\mathbf{U}$ & D & $\mathbf{U}$ & D & $\mathbf{U}$ & D & $\mathbf{U}$ & D & $\mathbf{U}$ & D & $\mathbf{U}$ & D & $\mathbf{U}$ & D & $\mathbf{U}$ \\
\hline D & 48 & 120 & 40 & 128 & 45 & 123 & 41 & 127 & 41 & 127 & 72 & 96 & $\mathbf{0}$ & 168 & 75 & 93 \\
\hline $\mathbf{U}$ & 55 & 164 & 41 & 178 & 52 & 167 & 55 & 164 & 55 & 164 & 96 & 123 & 0 & 219 & 116 & 103 \\
\hline Data as \% & \multicolumn{16}{|c|}{ Numbers are percent of column (predicted) total } \\
\hline D & 46.6 & 42.2 & 49.3 & 41.8 & 46.3 & 42.4 & 42.7 & 43.6 & 42.7 & 43.6 & 42.8 & 43.8 & $\mathbf{0 . 0 0}$ & 43.4 & 39.2 & 47.4 \\
\hline $\mathbf{U}$ & 53.4 & $\mathbf{5 7 . 7}$ & 50.6 & 58.1 & 53.6 & $\mathbf{5 7 . 5}$ & 57.2 & 56.3 & 57.2 & 56.3 & 57.1 & 56.1 & 0.00 & 56.5 & 60.7 & 52.5 \\
\hline
\end{tabular}

Finally, we approached forecasting using a decision tree methodology. A C5.0 Decision Tree was built using IBM's SPSS Modeler 14 package. The C5.0 algorithm is a technique often used in data mining for predicting or for classifying (Barry and Linoff, 2004). It requires a target variable that is non-numeric, and a set of input variables that may be either numeric or nonnumeric. In a step-wise process, the algorithm repeatedly separates the original data into smaller 
and smaller sets where each smaller set is more similar on the value of the target variable than was the immediately previous set. At each step of the process, the decision tree must decide which of the input variables does the best job splitting that set into more similar subsets. The variable chosen to use for splitting the data at each point is the one that provides the maximum information gain (Patil et al 2010). In other words, the algorithm checks each possible input variable at each step of the process. The one which would create a split with the most homogeneous groups following is the variable selected at that step. Various branches of the tree may use different splitting variables for their steps. The end result is a tree with an associated set of rules that classify any row of input data as likely to have a specific target value.

The decision tree for this problem was trained using the data up through December of 2009. We gave the decision tree the following inputs to use in building the tree: the up-down patterns from one to seven days, the number of up days in 1 to five days, and the closing value today. The resulting decision tree was wide and deep, and correct about $67 \%$ of the time. The results of the decision tree's accuracy for both the training and validation sets were calculated. These results are shown in the next three tables. Table 5 shows the overall results on the training data.

Table 5 Decision Tree results on training data

\begin{tabular}{|l|l|l|}
\hline Result & Count & Percent \\
\hline Correct & 10,165 & $67.38 \%$ \\
\hline Incorrect & 4,922 & $32.62 \%$ \\
\hline Total & 15,087 & \\
\hline
\end{tabular}

The results on the validation data are shown in Table 6. Here we see that the percent of correct forecasts for tomorrow's direction has dropped from the training set, but is still higher than the best result in Table 3 using the simple pattern forecasts. Thus, while the robustness from the training set shows a drop, the decision tree forecast remains a valuable model.

Table 6 Decision Tree results on validation data

\begin{tabular}{|l|l|l|}
\hline Result & Count & Percent \\
\hline Correct & 222 & $57.36 \%$ \\
\hline Incorrect & 165 & $42.64 \%$ \\
\hline Total & 387 & \\
\hline
\end{tabular}


We break this validation set data up further into correctness on each direction in the validation set, as shown in Table 7. This table shows both the number of times a forecast of up or down was made, and the percentage of times that the forecast was correct or incorrect. We see that the decision tree does a better job on Up forecasts than on Down, as have all the models. However, it is the only one of the forecasting methods to do better than $50 \%$ on Down days. Of the times that the decision tree predicted Up, it was correct $61 \%$ of the time, also a better performance than any of the simpler string methodologies.

Table 7 Matrix of Up and Down forecasts vs. actual movement

\begin{tabular}{|c|c|c|}
\hline & Forecasted Down & Forecasted Up \\
\hline Actual Down tomorrow & 72 & 96 \\
\hline & $51.06 \%$ & $39.02 \%$ \\
\hline Actual Up tomorrow & 69 & 150 \\
\hline & $48.94 \%$ & $60.98 \%$ \\
\hline
\end{tabular}

In addition to supplying a forecast for future values, this Modeler technique also looks at each of the input variables and ranks them in terms of relative importance to the model, with more important variables occurring higher up on the tree. The four variables ranked highest in importance to the forecast were the direction today, the closing value today, the 7-day pattern [for example UDUUDDU], and the number of Up movements in the last three days. Comparing this with the performance of the simple pattern forecasting models, we see that the simple pattern models that did the best were based on one-day and six-day patterns. The decision tree used oneday, seven-day and three-day information. So, both approaches took close and distant information into consideration.

\section{SUMMARY}

In this paper, we investigate the daily movement patterns from 1950 through 2011 for a total of 61 years, over 15 thousand observations. Our interest is in analyzing Up and Down movements independent of magnitude. We first look at the patterns created in this data set in two ways, as strings of Ups and Downs over time, and then as the number of Ups occurring with strings of different lengths. These values are tallied for each decade in order to discover whether or not specific patterns occur with the same frequency decade to decade. We find that, in every decade, 
there are more Up days than Down days, and the counts clearly show that some specific string patterns continue in about the same percent across time. This robustness in patterns across several decades is a useful finding that supports the longterm "buy and hold" strategies.

When we switch to counting the number of Up days within strings, regardless of where they occur, the patterns across decades become even more striking. In particular, we can see that 5-day combinations in every decade from 1950 on show that there are more strings with exactly 3 Ups than 2 Ups, more with 4 Ups than 1 Up, and more with 5 Ups than no Ups.

We use this pattern evidence to forecast future Up or Down movements in several ways, first by applying past pattern strings to future data, then by using the decision tree methodology. A C5.0 Decision Tree was built using IBM's SPSS Modeler 14 package. The C5.0 algorithm is a technique often used in data mining for prediction.

We find that the simple pattern forecasts, using only strings of one specific length at a time, do best with either one-day or six-day information. The decision tree outperforms these simple pattern forecasts by using information from multiple inputs with one-day, three-day, and seven-day patterns having the most influence. The ability of some of these models to generate forecasts which outperform the random walk gives us an indication that further research could improve the performance of models using the decision tree methodology employed in this paper to produce conditional forecasts. Further analysis needs to focus also on the optimal size of training and validation sets.

\section{References}

Allen F, Karjalainen R (1999) Using Genetic Algorithms to Find Technical Trading Rules. J Fin Econ 51:245-272

Avramov D, Chordia T (March 23, 2005). Predicting Stock Returns. Available at SSRN: http://ssrn.com/abstract=352980 or http://dx.doi.org/10.2139/ssrn.352980

Barberis N, Shleifer A, Vishny R (1998) A Model of Investor Sentiment. J Fin Econ 49:307-343

Baur D, Dimpfl T, Jung R (2012) Stock Return Autocorrelations Revisited: A Quantile Regression Approach. J of Emp Fin 19:251-265

Berry M, Linoff G (2004) Data Mining Techniques, Second Edition. Wiley Publishing, Inc., Indianapolis, Indiana 
Blume L, Easley D O'Hara M (1994) Market Statistics and Technical Analysis: The Role of Volume. J of Fin 49:153-181

Brock W, Lakonishok J, LeBaron B 91992) Simple Technical Trading Rules and the Stochastic Properties of Stock Returns. J of Fin 47:1731-1764

Brown D, Jennings R (1989) On Technical Analysis. The Rev of Fin Studies 2:527-551

Chan K, Chan KC, Karolyi GA (1991) Intraday Volatility in the Stock Index and Stock Index Futures Markets. Rev of Fin Studies 4:657-684

Cutler D, Poterba J, Summers L (1991) Speculative Dynamics. Rev of Econ Studies 58:529-546

Daniel K, Hirshleifer D, Subrahmanyam A (1998) Investor Psychology and Security Market Under- and Overreactions. J of Fin 52:1839-1885

DeLong J, Shleifer A, Summers L, Waldmann R (1990) Positive Feedback Investment Strategies and Destabilizing Rational Speculation. 45:379-395

Doran J, Ronn E, Goldberg R (November 10, 2009) A Simple Model for Time - Varying Expected Returns on the S\&P 500 Index Journal of Investment Management, Second Quarter 2009. Available at SSRN: http://ssrn.com/abstract $=1503581$

Fama E, Blume M (1966) Filter Rules and Stock-Market Trading. J of Bus 39:226-241

Gencay R 91998) The Predictability of Security Returns with Simple Technical Trading Rules. J of Empirical Fin 5:347-359.

Hong H, Stein J (1999) A Unified Theory of Underreaction, Momentum Trading, and Overreaction in Asset markets. J of Fin 54:2143-2184

Jensen M, Benington G (1970) Random Walks and Technical Theories: Some Additional Evidence. J of Fin 25:469-482

Lo A, Mamaysky H, Wang J (2000) Foundations of Technical Analysis: Computational Algorithms, Statistical Inference, and Empirical Implementation. J of Fin 55:1705-1765

McInish T, Wood R (1992) An Analysis of Intraday Patterns in Bid/Ask Spreads for NYSE Stocks. J of Fin 47:753-764

Patil N, Lathi R, Chitre V (2012) Comparison of C5.0 \& CART Classification algorithms using pruning technique. Int J Eng Res \& Technol 1(4):1-5 
Schulmeister S (March 1, 2007) The Profitability of Technical Stock Trading has Moved from Daily to Intraday Data. WIFO Working Paper No. 289. Available at SSRN: http://ssrn.com/abstract=1714980 or http://dx.doi.org/10.2139/ssrn. 1714980 